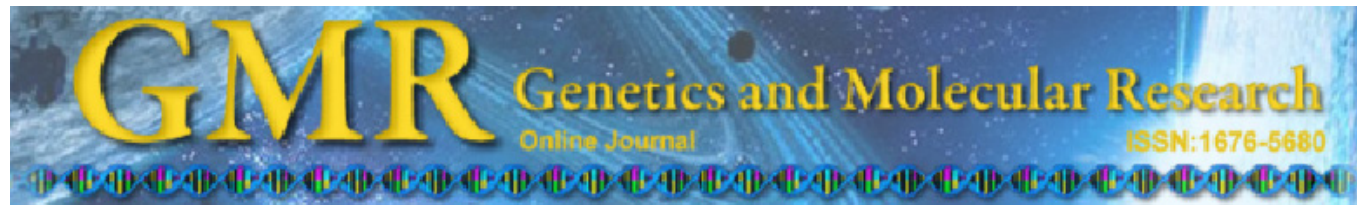

\title{
Association between interleukin 21 and Graves' disease
}

\author{
H.Y. Jia ${ }^{1 *}$, Z.G. Zhang ${ }^{1 *}$, X.J. Gu ${ }^{3}$, T. Guo ${ }^{1,2}$, B. Cui ${ }^{1,2}$, G. Ning ${ }^{1,2}$ and Y.J. Zhao ${ }^{1}$ \\ ${ }^{1}$ Shanghai Clinical Center for Endocrine and Metabolic Diseases, \\ Shanghai Institute of Endocrine and Metabolic Diseases, Ruijin Hospital, \\ Shanghai JiaoTong University School of Medicine, Shanghai, China \\ ${ }^{2}$ Laboratory Endocrine \& Metabolic Diseases of Institute of Health Sciences, \\ Shanghai Institutes for Biological Sciences, \\ Chinese Academy of Sciences and Shanghai JiaoTong University School of Medicine, \\ Shanghai, China \\ ${ }^{3}$ Department of Endocrine and Metabolic Diseases, \\ The First Affiliated Hospital of Wenzhou Medical College, Wenzhou, China \\ *These authors contributed equally to this study. \\ Corresponding author: Y.J. Zhao \\ E-mail: yongju-zhao@hotmail.com
}

Genet. Mol. Res. 10 (4): 3338-3346 (2011)

Received March 25, 2011

Accepted September 7, 2011

Published October 31, 2011

DOI http://dx.doi.org/10.4238/2011.October.31.6

ABSTRACT. Graves' disease (GD) is an organ-specific autoimmune thy-
roid disease; $25-50 \%$ of GD patients will develop Graves' ophthalmopathy
(GO). The etiology of GD and GO may be multifactorial, but the immune
response plays a central role. Many studies have reported that IL- 21 has
crucial roles in autoimmune diseases. We examined whether IL- 21 is as-
sociated with the development of GD and GO. The serum concentration of
IL- 21 was tested in 40 primary GD patients, 42 treated GO patients and 24
healthy controls. Our data show that the serum level of IL-21 is associated
with the development of GD. We also made an association study with the
IL-21 gene polymorphisms rs 4833837 , rs 907715 and rs 13143866 in a com-
parison of 633 patients and 612 healthy controls from the Chinese popula-
tion. This case-control association study demonstrated that rs907715 SNP is
significantly associated with GD, while the rs13143866 A allele is signifi-
cantly associated with GO. The haplotypes A-G-G and A-A-A were found 
at higher and lower frequencies, respectively, in GD patients, suggesting a protective role for A-A-A. However, there were no significant differences in the frequencies of these haplotypes between the GO patients and the control group. We found no association between IL-21 gene polymorphisms and the age of GD onset. We conclude that IL-21 is associated with GD and GO.

Key words: IL-21; Graves' disease; Graves'ophthalmopathy

\section{INTRODUCTION}

Graves' disease (GD) is an organ-specific autoimmune thyroid disease and characterized by the presence of thyroid-specific reactive T lymphocytes and autoantibodies and typical clinical manifestations (Ban and Tomer, 2003). About 25-50\% individuals with GD have clinical manifestation of Graves' ophthalmopathy (GO) (Bahn, 1995). Infiltration of activated T cells, B cells, and macrophages as well as the expansion of eye muscles and orbital fat tissues can be observed through histologic examinations of the retrobulbar tissues (Weetman et al., 1989). The course of GO is unpredictable and rapid deterioration can occur at any time (Bednarczuk et al., 2007; Yamada et al., 2000). The etiology of GD and GO may be multifactorial, involving complex interactions between genetic, environmental, endogenous and local factors (Farid and Balazs, 1998). However, immune response plays a central role in the pathogenesis of GD. Cytokines, a large group of non-enzymatic proteins, participate in the induction and effector phases of all inflammatory and immune responses, and are therefore likely to play a critical role in the development of autoimmune diseases (Chen et al., 2005).

Liu et al. (2008) have determined that the interleukin 21 (IL-21) gene is located at chromosome 4q27, and it was recently shown to be associated with four autoimmune diseases (Celiac disease, type 1 diabetes, Grave's disease and rheumatoid arthritis). IL-21, a newly identified member of the $\gamma$-chain family, was originally thought to be produced only by CD4+ T cells, but it is also produced by Th17 cells and NKT cells, indicating its role both in innate and adaptive immune responses (Spolski and Leonard, 2008). It signals through the common $\gamma$ - chain of the IL-2 receptor and its own unique receptor (designated IL-21R) (Leonard and Spolski, 2005). IL-21R, consisting of a unique IL-21R-a chain and the common $\gamma$-chain, is widely expressed on T cells, B cells, NK cells, and dendritic cells in all lymphoid tissues, as well as some nonimmune cells, such as fibroblasts and epithelial cells (Davis et al., 2007). IL-21 can therefore exert a variety of regulatory effects on both lymphoid and nonlymphoid cells (Caprioli et al., 2008). In the last few years, it has become clear that not only does IL-21 regulate normal lymphoid development and function, it also serves crucial roles in inflammatory responses, allergy, autoimmunity, and certain antitumor actions (Spolski and Leonard, 2008). High production of IL-21 has been described in various immune-mediated diseases, including inflammatory bowel diseases, celiac disease, and Helicobacter pylori-related gastritis (Caruso et al., 2007). Previous studies have shown that the disruption of the IL-21 gene or injection of recombinant IL-21 enhances the expression of experimental autoimmune myasthenia gravis (EAMG) and experimental autoimmune encephalomyelitis (EAE), respectively (Liu et al., 2006; Vollmer et al., 2005). It has been suggested that the manipulation of IL-21-mediated signaling directly alters the function of CD8 + cells, B cells, Thelper cells, and NK cells In these experimental systems (Piao et al., 2008).

The $I L-21$ gene, located at chromosome 4q26-q27, consists of 5 exons spanning approximately $8.44 \mathrm{~kb}$ of genomic DNA. To understand how IL-21 is involved in the develop- 
ment of the disorder, we determined the serum levels of IL-21 in Chinese patients with GD and/or GO and controls. We also performed an association study with the $I L-21$ gene polymorphisms between 633 patients and 612 healthy controls. Our results showed that interleukin-21 was strongly associated with GD and GO.

\section{SUBJECTS AND METHODS}

\section{Subjects}

GD patients ( $\mathrm{N}=633,158$ males and 475 females, aged 10-81 years, mean age of $38.9 \pm$ 13.7 years) being treated at Ruijin Hospital were enrolled in this study. GD was diagnosed on the basis of clinical manifestations, biochemical criteria of thyrotoxicosis $(\mathrm{TSH}<0.05 \mathrm{mIU} / \mathrm{L}$ and increased free T3 and/or free T4), and the presence of TSH receptor antibodies (Meller et al., 2000). GO was diagnosed by experienced ophthalmologists and was classified using the "NOSPECS" classification (Mourits et al., 1989; Lin et al., 2008); patients with class 2-6 were considered as having GO. There were 171 GD patients with vert-ophthalmopathy (NOSPECS class 2 or more; GO) and 462 GD patients without or with mild ophthalmopathy (NOSPECS class 0 and 1).

Healthy Chinese volunteers $(\mathrm{N}=612,145$ males and 467 females, aged 17-85 years, mean age of $51.1 \pm 15.0$ years) from the same region of China without family history of GD or other autoimmune diseases served as the control group. Informed consent was obtained from all participants, and the study was approved by the Ethics Committee of Ruijin Hospital, Shanghai JiaoTong University, School of Medicine.

\section{Measurement of serum IL-21 concentration}

The concentration of IL-21 polypeptide in the different sera was determined with a commercially available ELISA kit (R\&D Systems, Minneapolis, MN). The following sera were tested: 40 sera of primary GD patients, 42 sera of treated active GO patients and 24 sera of healthy controls.

\section{$I L-21$ gene SNP selection}

For the selection of the SNPs, Haploview software (http://www.broad.mit.edu/mpg/ haploview) was applied to perform linkage disequilibrium and haplotype block analyses using Hapmap phase genotype data for the chromosomal region 4:123,750,234..123,764,662 (CHB database, Hapmap release 27). The amplicon of interest was a $14.4 \mathrm{~kb}$ region, with the $I L-21$ gene and approximately $3 \mathrm{~kb}$ upstream and $3 \mathrm{~kb}$ downstream of the $I L-21$ gene. The selection of tag-SNP was performed by running the tagger program implemented in Haploview (Barrett et al., 2005). The criterion for $\mathrm{r}^{2}$ was set at $>0.8$. Four tag-SNPs (rs2221903, rs4833837, rs907715, rs 13143866) were chosen. The two SNPs of rs2221903 and rs907715 were analyzed in linkage disequilibrium $\left(\mathrm{r}^{2}=1\right)$, and $\mathrm{rs} 907715$ was chosen in our study.

\section{Genotyping}

Genomic DNA from peripheral blood was extracted using a commercially available kit (Qiagen, Hilden, Germany) according to the manufacturer instruction. The four tag-SNPs were 
genotyped on the GenomeLab SNPstream 12-plex Genotyping System (Beckman \& Coulter Fullerton, CA, USA) following manufacturer instructions. This platform uses a single-base pair extension reaction to incorporate two-color fluorescence terminal nucleotides that are detected by a specialized imager (Liang et al., 2008). To confirm the results of genotyping by the SNPstream System, 100 random samples were selected to perform a direct sequencing analysis, and the results of both methods were identical.

\section{Statistics}

The genotype distributions in both groups (control and GD) were examined using Hardy-Weinberg equilibrium. Clinical data were expressed as mean $\pm \mathrm{SD}$. The difference in IL-21 serum concentrations was calculated using the independent samples t-test. Allelic association for individual IL-21 SNPs with GD was performed by the $\chi^{2}$ test or Fisher's exact test within the SPSS for Windows version 11.0 statistical package (SPSS, Chicago, IL, USA). The strength of the association between the presence of the disease and the constitutionally determined alleles was estimated by the odds ratio (OR). OR and $95 \%$ confidence intervals (CIs) were calculated using the Mantel-Haenszel method. The associations of genotype and frequencies with GD were estimated using the SNPStats program (Solé et al., 2006) separately under the additive, dominant, and recessive model. The program SNPStats was also adopted for haplotype analysis. In a first approximation, all haplotypes identified were computed, but in order to minimize loss of power, haplotypes with a frequency lower than $5 \%$ were excluded from further analysis.

\section{RESULTS}

\section{The different serum levels of IL-21 between patients and normal controls}

The serum concentration of IL-21 in the primary GD patients was significantly higher than that of the healthy controls $(\mathrm{P}=0.001)$ and treated active $\mathrm{GO}$ patients $(\mathrm{P}=0.008)$. The mean serum concentration of IL-21 in treated active GO patients was also higher than that of the healthy controls, $95.0 \mathrm{vs} 87.2 \mathrm{pg} / \mathrm{mL}$; however, there was no statistically significant difference $(\mathrm{P}>0.05)$ (Figure 1).

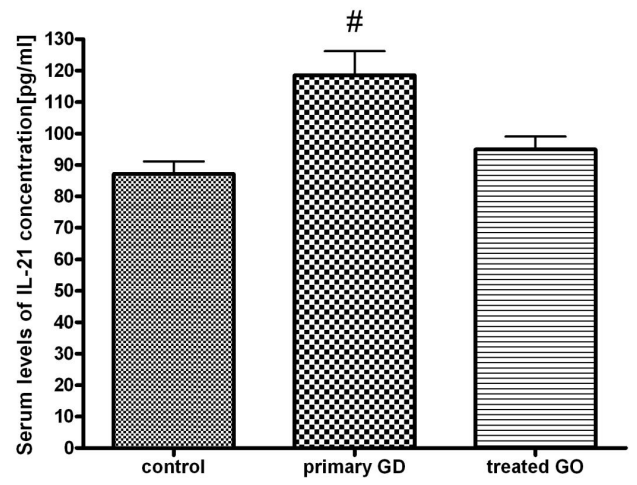

Figure 1. Serum levels of IL-21 in primary GD patients, treated active GO patients and normal controls. \# stands for the significant difference between the primary GD patients and normal controls. 


\section{Table 1A. IL-21 gene variations and associations with GD.}

\begin{tabular}{llll}
\hline IL-21 gene polymorphism & GD $(\%)$ & Control subjects $(\%)$ & $\chi^{2}$-test P-value \\
\hline rs4833837(598/618) & & & \\
Genotype frequencies & & $453(75.8)$ & $\chi^{2}=1.79$ \\
AA & $488(79)$ & $138(23.1)+7(1.1)$ & $P_{\text {dominant }}=0.18$ \\
AG+GG & $125(20.2)+5(0.8)$ & $1044(87.3)$ & $\chi^{2}=1.86$ \\
Allele frequencies & $1101(89.1)$ & $152(12.7)$ & $\mathrm{P}=0.172$ \\
A & $135(10.9)$ & & $\chi^{2}=18.37$ \\
G & & $180(30)$ & $P_{\text {dominant }}=2 \times 10^{-5}, P c=6 \times 10^{-5}$ \\
rs907715(600/618) & $120(19.4)$ & $284(47.3)+136(22.7)$ & $\chi^{2}=16.05$ \\
Genotype frequencies & $323(52.3)+175(28.3)$ & $644(53.7)$ & $\mathrm{P}=6 \times 10^{-5}, P c=1.8 \times 10^{-4}$ \\
AA & $563(45.6)$ & $556(46.3)$ & \\
AG+GG & $673(54.4)$ & & $\chi^{2}=3.20$ \\
Allele frequencies & & $501(83.9)$ & $P_{\text {dominant }}=0.073$ \\
A & & $88(14.7)+8(1.3)$ & $\chi^{2}=3.30$ \\
G & $495(80)$ & $1090(91.3)$ & $\mathrm{P}=0.069$ \\
rs13143866(597/619) & $113(18.2)+11(1.8)$ & $104(8.7)$ & \\
Genotype frequencies & $1103(89.1)$ & & \\
GG & $135(10.9)$ & & \\
AG+AA & & & \\
Allele frequencies & & & \\
A & & & \\
\hline
\end{tabular}

Table 1B. IL-21 gene variations and associations with GO.

\begin{tabular}{|c|c|c|c|}
\hline IL-21 gene polymorphism & GO (\%) & Control subjects (\%) & $\chi^{2}$-test P-value \\
\hline \multicolumn{4}{|l|}{ rs4833837(598/164) } \\
\hline \multicolumn{4}{|l|}{ Genotype frequencies } \\
\hline $\mathrm{AA}$ & $134(81.7)$ & $453(75.8)$ & $\chi^{2}=2.58$ \\
\hline $\mathrm{AG}+\mathrm{GG}$ & $30(18.3)+0(0)$ & $138(23.1)+7(1.1)$ & $P_{\text {dominant }}=0.108$ \\
\hline \multicolumn{4}{|l|}{ Allele frequencies } \\
\hline A & $298(90.9)$ & $1044(87.3)$ & $\chi^{2}=3.11$ \\
\hline G & $30(9.1)$ & $152(12.7)$ & $\mathrm{P}=0.078$ \\
\hline \multicolumn{4}{|l|}{ rs907715(600/165) } \\
\hline \multicolumn{4}{|l|}{ Genotype frequencies } \\
\hline $\mathrm{AA}$ & $32(19.4)$ & $180(30)$ & $\chi^{2}=7.27$ \\
\hline $\mathrm{AG}+\mathrm{GG}$ & $93(56.4)+40(24.2)$ & $284(47.3)+136(22.7)$ & $\stackrel{P}{\text { dominant }}=0.007, P c=0.021$ \\
\hline \multicolumn{4}{|l|}{ Allele frequencies } \\
\hline A & $157(47.6)$ & $644(53.7)$ & $\chi^{2}=3.85$ \\
\hline G & $173(52.4)$ & $556(46.3)$ & $\mathrm{P}=0.04976, P c=0.14928$ \\
\hline \multicolumn{4}{|l|}{ rs13143866(597/161) } \\
\hline \multicolumn{4}{|l|}{ Genotype frequencies } \\
\hline GG & $109(67.7)$ & $501(83.9)$ & $\chi^{2}=21.23$ \\
\hline $\mathrm{AG}+\mathrm{AA}$ & $50(31.1)+2(1.2)$ & $88(14.7)+8(1.3)$ & $P_{\text {dominant }}=4 \times 10^{-6}, P c=1.2 \times 10^{-5}$ \\
\hline \multicolumn{4}{|l|}{ Allele frequencies } \\
\hline $\mathrm{G}$ & $268(83.2)$ & $1090(91.3)$ & $\chi^{2}=17.65$ \\
\hline A & $54(16.8)$ & $104(8.7)$ & $\stackrel{n}{\mathrm{P}}=3 \times 10^{-5}, P c=9 \times 10^{-5}$ \\
\hline
\end{tabular}

\section{Single variant association}

Three SNPs were identified in this study, and all were in Hardy-Weinberg equilibrium. Table 1A shows the complete genotype and allele frequency data. There was evidence that 
carriers of the rs907715 G allele had increased susceptibility to GD. A significantly higher percentage of GD patients had the G allele compared to the control subjects at an even lower level of P-value $\left[P_{\text {allele }}=6 \mathrm{E}-05, P_{c}=1.8 \mathrm{E}-04\right]$. The SNP rs 13143866 A allele was significantly associated with GO $\left[P_{\text {allele }}=3 \mathrm{E}-5, P_{\mathrm{c}}=9 \mathrm{E}-5\right]$, but not with GD.

\section{Haplotype analysis}

Haplotype analyses were performed using the SNPStats program. Rare haplotypes (frequencies $<0.05$ ) were combined. The most common haplotype $(A-A-G)$ served as the reference haplotype in our analysis (Tables 2A,B). There was a significant difference in the global haplotype profile between the GD and control group $(\mathrm{P}<0.0001)$. Compared to the controls, the haplotype A-G-G showed higher frequency in GD patients (39 vs $34 \% ; \mathrm{P}=0.012, P_{\mathrm{c}}=$ $0.036 ; \mathrm{OR}=1.28,95 \% \mathrm{CI}=1.06-1.55)$. The haplotype A-A-A had a lower frequency in GD patients $\left(5 v s 9 \% ; \mathrm{P}=0.0062, P_{\mathrm{c}}=0.019 ; \mathrm{OR}=0.55,95 \% \mathrm{CI}=0.36-0.84\right)$. This suggests that the haplotype A-G-G may play a harmful role, while the haplotype A-A-A may exert a protective effect. However, there was no significant difference between the GO patients and the controls.

Table 2A. Three marker haplotype frequencies in a case-control cohort of patients with GD.
\begin{tabular}{lcccccccc}
\hline No. & rs4833837 & rs907715 & rs13143866 & Controls/cases & P -value $\left(\mathrm{P}_{\mathrm{c}}\right)$ & OR & $95 \%$ CI \\
\hline 1 & $\mathrm{~A}$ & $\mathrm{~A}$ & $\mathrm{G}$ & $0.45 / 0.41$ & & 1 & \\
2 & $\mathrm{~A}$ & $\mathrm{G}$ & $\mathrm{G}$ & $0.34 / 0.39$ & $0.012(0.036)$ & 1.28 & $(1.06-1.55)$ \\
3 & $\mathrm{G}$ & $\mathrm{G}$ & $\mathrm{G}$ & $0.12 / 0.09$ & 0.088 & 0.78 & $(0.58-1.04)$ \\
4 & $\mathrm{~A}$ & $\mathrm{~A}$ & $\mathrm{~A}$ & $0.09 / 0.05$ & $0.0062(0.019)$ & 0.55 & $(0.36-0.84)$ \\
rare & $*$ & $*$ & $*$ & $0.06 / 0.001$ & $<0.0001$ & 72.06 & $(9.05-546.81)$ \\
\hline
\end{tabular}

Global haplotype association P-value: $<0.0001$.

Table 2B. Three marker haplotype frequencies in a case-control cohort of patients with GO.

\begin{tabular}{lccccccc}
\hline No. & rs4833837 & rs907715 & rs13143866 & Control/case & P -value $\left(\mathrm{P}_{\mathrm{c}}\right)$ & OR & $95 \%$ CI \\
\hline 1 & $\mathrm{~A}$ & $\mathrm{~A}$ & $\mathrm{G}$ & $0.45 / 0.40$ & & 1 & $(1.39$ \\
2 & $\mathrm{~A}$ & $\mathrm{G}$ & $\mathrm{G}$ & $0.34 / 0.38$ & $0.047(0.141)$ & $0.1 .93)$ \\
3 & $\mathrm{G}$ & $\mathrm{G}$ & $\mathrm{G}$ & $0.12 / 0.06$ & $0.049(0.147)$ & 0.50 & $(0.25-1.00)$ \\
4 & $\mathrm{~A}$ & $\mathrm{~A}$ & $\mathrm{~A}$ & $0.09 / 0.08$ & 0.64 & 1.15 & $(0.63-2.11)$ \\
Rare & $*$ & $*$ & $*$ & $0 / 0.09$ & $<0.0001$ & 117.45 & $(15.18-908.72)$ \\
\hline
\end{tabular}

Global haplotype association P-value: $<0.0001 . \mathrm{GO}=$ Graves' disease associated ophthalmopathy; $\mathrm{OR}=$ odds ratio; $\mathrm{CI}=$ confidence interval. The most common haplotype is reference. Data are presented as frequency. $\mathrm{P}$ values $<0.05$ are considered significant. ${ }^{a}$ Rare haplotype combined. Each haplotype has a frequency below $5 \%$ among the controls.

\section{Association between $I L-21$ gene polymorphisms and the age of GD onset}

Both the G-allele and GG genotype frequencies of rs13143866 in the subjects with an earlier onset age of GD ( $<40$ years $)$ appeared greater than that in the subjects with a later onset ( $\geq 40$ years), but there was no statistically significant difference $\left(P_{\text {allele }}=0.032, P_{\mathrm{c}}=\right.$ 0.096; $P_{\text {dominant }}=0.045, P_{\mathrm{c}}=0.135$; Tables 3A). The frequency of haplotype A-G-A was higher in the subjects with a later onset age of GD than that in the subjects with an earlier onset, but there was no statistically significant difference ( 7 v $3 \%$; $\mathrm{P}=0.036, P_{\mathrm{c}}=0.108$; $\mathrm{OR}=2.06 ; 95 \% \mathrm{CI}=1.05-4.04)($ Table $3 \mathrm{~B})$. 


\begin{tabular}{|c|c|c|c|c|c|c|c|}
\hline \multicolumn{2}{|l|}{$\begin{array}{l}\text { IL-21 gene } \\
\text { polymorphism }\end{array}$} & \multicolumn{2}{|c|}{$<40$ years } & \multicolumn{2}{|c|}{$\geq 40$ years } & \multicolumn{2}{|c|}{$\chi^{2}$-test P-value } \\
\hline \multicolumn{8}{|c|}{ rs $4833837(376 / 242)$} \\
\hline \multicolumn{8}{|c|}{ Genotype frequencies } \\
\hline AA & & \multirow{2}{*}{\multicolumn{2}{|c|}{$\begin{array}{c}300(79.8) \\
72(19.1)+4(1.1)\end{array}$}} & \multicolumn{2}{|c|}{$188(77.7)$} & \multirow{3}{*}{\multicolumn{2}{|c|}{$\begin{array}{c}\chi^{2}=0.39 \\
P_{\text {dominant }}=0.532\end{array}$}} \\
\hline $\mathrm{AG}+\mathrm{GG}$ & & & & $53(21.9)$ & $0.4)$ & & \\
\hline \multicolumn{6}{|c|}{ Allele frequencies } & & \\
\hline A & & \multicolumn{2}{|c|}{$672(89.4)$} & \multicolumn{2}{|c|}{$429(88.6)$} & \multirow{2}{*}{\multicolumn{2}{|c|}{$\begin{array}{l}\chi^{2}=0.16 \\
P=0.690\end{array}$}} \\
\hline $\mathrm{G}$ & & \multicolumn{2}{|c|}{$80(10.6)$} & \multicolumn{2}{|c|}{$55(11.4)$} & & \\
\hline \multicolumn{8}{|c|}{ rs $907715(375 / 243)$} \\
\hline \multicolumn{8}{|c|}{ Genotype frequencies } \\
\hline $\mathrm{AA}$ & & \multirow{2}{*}{\multicolumn{2}{|c|}{$\begin{array}{c}109(29) \\
193(51.5)+73(19.5)\end{array}$}} & \multirow{2}{*}{\multicolumn{2}{|c|}{$\begin{array}{c}66(27.2) \\
130(53.5)+47(19.3)\end{array}$}} & \multirow{2}{*}{\multicolumn{2}{|c|}{$\begin{array}{c}\chi^{2}=0.26 \\
P_{\text {dominant }}=0.607\end{array}$}} \\
\hline $\mathrm{AG}+\mathrm{GG}$ & & & & & & & \\
\hline \multicolumn{8}{|c|}{ Allele frequencies } \\
\hline G & & \multicolumn{2}{|c|}{$411(54.8)$} & \multicolumn{2}{|c|}{$262(53.9)$} & \multirow{2}{*}{\multicolumn{2}{|c|}{$\begin{array}{l}\chi^{2}=0.09 \\
P=0.759\end{array}$}} \\
\hline $\mathrm{A}$ & & \multicolumn{2}{|c|}{$339(45.2)$} & \multicolumn{2}{|c|}{$224(46.1)$} & & \\
\hline \multicolumn{8}{|c|}{ rs13143866(378/241) } \\
\hline \multicolumn{8}{|c|}{ Genotype frequencies } \\
\hline GG & & \multirow{3}{*}{\multicolumn{2}{|c|}{$\begin{array}{c}312(82.5) \\
61(16.1)+5(1.3)\end{array}$}} & \multirow{3}{*}{\multicolumn{2}{|c|}{$\begin{array}{c}183(75.9) \\
52(21.6)+6(2.5)\end{array}$}} & & $=4.01$ \\
\hline $\mathrm{AG}+\mathrm{AA}$ & & & & & & $P_{\text {dominant }}$ & $45, P c=0.135$ \\
\hline Allele frequenc & & & & & & & \\
\hline G & & 685 & & 418 & & & $=4.58$ \\
\hline A & & 71 & & 64( & & $P=($ & $P c=0.096$ \\
\hline No. & rs4833837 & rs907715 & rs 13143866 & $<40 / \geq 40$ & $\mathrm{P}$-value & OR & $95 \% \mathrm{CI}$ \\
\hline 1 & $\mathrm{~A}$ & A & $\mathrm{G}$ & $0.41 / 0.41$ & 1 & & \\
\hline 2 & A & G & G & $0.41 / 0.35$ & 0.29 & 0.86 & $(0.65-1.13)$ \\
\hline 3 & G & G & G & $0.09 / 0.10$ & 0.71 & 1.09 & $(0.69-1.72)$ \\
\hline 4 & A & G & A & $0.03 / 0.07$ & 0.036 & 2.06 & $(1.05-4.04)$ \\
\hline 5 & A & A & A & $0.04 / 0.05$ & 0.91 & 1.04 & $(0.48-2.25)$ \\
\hline 6 & G & G & A & $0.02 / 0.01$ & 0.9 & 0.92 & $(0.24-3.55)$ \\
\hline rare & $*$ & $*$ & $*$ & $0.002 / 0$ & 1 & 0.00 & (-Inf-Inf) \\
\hline
\end{tabular}

Global haplotype association P-value: 0.2.

\section{DISCUSSION}

IL-21 as a cytokine affects the function of a number of immune competent cells upon binding to its receptor (IL-21R) (Mehta et al., 2004). The effects of IL-21 on effector cells are pleomorphic, as it has been shown to both promote and suppress immune responses. IL-21 augments the proliferation of CD4+ and CD8+ T lymphocytes, regulates the survival and the profile of cytokines secreted by these cells, drives the differentiation of B cells into memory cells and terminally differentiated plasma cells, and enhances the activity of NK cells (ParrishNovak et al., 2000; Kasaian et al., 2002; Ozaki et al., 2002; Strengell et al., 2003).

GD is a T helper 2 (Th2) predominant autoimmune disorder characterized by the presence of anti-thyrotropin receptor antibody, which stimulates thyroid hormone secretion (Hiromatsu et al., 2006). Thyroid-associated ophthalmopathy is generally considered to be an autoimmune disorder that is closely associated with GD (Wall et al., 1991). However, only some GD patients develop clinically evident ophthalmopathy. The mechanisms of the development of ophthalmopathy are still unclear. Cytokines from infiltrating lymphocytes and macrophages may serve important functions in the initiation and propagation of the autoimmune process in the orbit (Hiromatsu et al., 2002). 
In the present study, we found that the serum IL-21 levels of GD patients were significantly higher than that of the healthy controls. This suggests that IL-21 could play a role in the pathogenesis of GD. However, there was no significant difference in serum IL-21 levels between treated active GO patients and the healthy controls; drug intervention in GO patients could have impacted the results. We also investigated $I L-21$ gene polymorphism for the risk of GD in Chinese GD and GO patients. The case-control association study results showed that the SNP rs907715 was significantly associated with GD and that the rs13143866 A allele was significantly associated with GO patients.

The haplotype A-G-G showed higher frequency in GD patients and the haplotype AA-A had a lower frequency in GD patients. This suggests that the haplotype A-G-G may play a harmful role, and that the haplotype A-A-A may exert a protective effect. Nevertheless, there was no significant difference between the GO patients and the controls. There was no association between IL-21 gene polymorphisms and the age of GD onset. In conclusion, IL-21 gene may have an important effect during the development of GD and GO.

\section{ACKNOWLEDGMENTS}

Research supported by Chinese National Natural Science Foundation (\#30800538 and \#81070635), Wenzhou Science and Technology Project (\#Y20090038). The present study would not have been possible without the participation of the patients and healthy volunteers.

\section{REFERENCES}

Bahn RS (1995). Assessment and management of the patient with Graves' ophthalmopathy. Endocr. Pract. 1: 172-178.

Ban Y and Tomer Y (2003). The contribution of immune regulatory and thyroid specific genes to the etiology of Graves' and Hashimoto's diseases. Autoimmunity 36: 367-379.

Barrett JC, Fry B, Maller J and Daly MJ (2005). Haploview: analysis and visualization of LD and haplotype maps. Bioinformatics 21: 263-265.

Bednarczuk T, Gopinath B, Ploski R and Wall JR (2007). Susceptibility genes in Graves' ophthalmopathy: searching for a needle in a haystack? Clin. Endocrinol. 67: 3-19.

Caprioli F, Sarra M, Caruso R, Stolfi C, et al. (2008). Autocrine regulation of IL-21 production in human T lymphocytes. J. Immunol. 180: 1800-1807.

Caruso R, Fina D, Peluso I, Fantini MC, et al. (2007). IL-21 is highly produced in Helicobacter pylori-infected gastric mucosa and promotes gelatinases synthesis. J. Immunol. 178: 5957-5965.

Chen RH, Chen WC, Chang CT, Tsai CH, et al. (2005). Interleukin-1-beta gene, but not the interleukin-1 receptor antagonist gene, is associated with Graves' disease. J. Clin. Lab. Anal. 19: 133-138.

Davis ID, Skak K, Smyth MJ, Kristjansen PE, et al. (2007). Interleukin-21 signaling: functions in cancer and autoimmunity. Clin. Cancer Res. 13: 6926-6932.

Farid NR and Balazs C (1998). The genetics of thyroid associated ophthalmopathy. Thyroid 8: 407-409.

Hiromatsu Y, Kaku H, Miyake I, Murayama S, et al. (2002). Role of cytokines in the pathogenesis of thyroid-associated ophthalmopathy. Thyroid 12: 217-221.

Hiromatsu Y, Mukai T, Kaku H, Miyake I, et al. (2006). IL-18 gene polymorphism confers susceptibility to the development of anti-GAD65 antibody in Graves' disease. Diabet. Med. 23: 211-215.

Kasaian MT, Whitters MJ, Carter LL, Lowe LD, et al. (2002). IL-21 limits NK cell responses and promotes antigenspecific T cell activation: a mediator of the transition from innate to adaptive immunity. Immunity 16: 559-569.

Leonard WJ and Spolski R (2005). Interleukin-21: a modulator of lymphoid proliferation, apoptosis and differentiation. Nat. Rev. Immunol. 5: 688-698.

Liang J, Chen P, Hu Z, Zhou X, et al. (2008). Genetic variants in fibroblast growth factor receptor 2 (FGFR2) contribute to susceptibility of breast cancer in Chinese women. Carcinogenesis 29: 2341-2346.

Lin MC, Hsu FM, Bee YS and Ger LP (2008). Age influences the severity of Graves' ophthalmopathy. Kaohsiung J. Med. 
Sci. 24: 283-288.

Liu R, Van KL, La CA, Price M, et al. (2006). Autoreactive T cells mediate NK cell degeneration in autoimmune disease. J. Immunol. 176: 5247-5254.

Liu Y, Helms C, Liao W, Zaba LC, et al. (2008). A genome-wide association study of psoriasis and psoriatic arthritis identifies new disease loci. PLoS Genet. 4: e1000041.

Mehta DS, Wurster AL and Grusby MJ (2004). Biology of IL-21 and the IL-21 receptor. Immunol. Rev. 202: 84-95.

Meller J, Jauho A, Hufner M, Gratz S, et al. (2000). Disseminated thyroid autonomy or Graves' disease: reevaluation by a second generation TSH receptor antibody assay. Thyroid 10: 1073-1079.

Mourits MP, Koornneef L, Wiersinga WM, Prummel MF, et al. (1989). Clinical criteria for the assessment of disease activity in Graves' ophthalmopathy: a novel approach. Br. J. Ophthalmol. 73: 639-644.

Ozaki K, Spolski R, Feng CG, Qi CF, et al. (2002). A critical role for IL-21 in regulating immunoglobulin production. Science 298: 1630-1634.

Parrish-Novak J, Dillon SR, Nelson A, Hammond A, et al. (2000). Interleukin 21 and its receptor are involved in NK cell expansion and regulation of lymphocyte function. Nature 408: 57-63.

Piao WH, Jee YH, Liu RL, Coons SW, et al. (2008). IL-21 modulates CD4+ CD25+ regulatory T-cell homeostasis in experimental autoimmune encephalomyelitis. Scand. J. Immunol. 67: 37-46.

Solé X, Guinó E, Valls J, Iniesta R, et al. (2006). SNPStats: a web tool for the analysis of association studies. Bioinformatics 22: 1928-1929.

Spolski R and Leonard WJ (2008a). Interleukin-21: basic biology and implications for cancer and autoimmunity. Annu. Rev. Immunol. 26: 57-79.

Spolski R and Leonard WJ (2008b). The Yin and Yang of interleukin-21 in allergy, autoimmunity and cancer. Curr. Opin. Immunol. 20: 295-301.

Strengell M, Matikainen S, Siren J, Lehtonen A, et al. (2003). IL-21 in synergy with IL-15 or IL-18 enhances IFN-gamma production in human NK and T cells. J. Immunol. 170: 5464-5469.

Vollmer TL, Liu R, Price M, Rhodes S, et al. (2005). Differential effects of IL-21 during initiation and progression of autoimmunity against neuroantigen. J. Immunol. 174: 2696-2701.

Wall JR, Salvi M, Bernard NF, Boucher A, et al. (1991). Thyroid-associated ophthalmopathy - a model for the association of organ-specific autoimmune disorders. Immunol. Today 12: 150-153.

Weetman AP, Cohen S, Gatter KC, Fells P, et al. (1989). Immunohistochemical analysis of the retrobulbar tissues in Graves' ophthalmopathy. Clin. Exp. Immunol. 75: 222-227.

Yamada M, Li AW and Wall JR (2000). Thyroid-associated ophthalmopathy: clinical features, pathogenesis, and management. Crit. Rev. Clin. Lab. Sci. 37: 523-549. 